\section{Zur Isolierung neuer in der Natur vorkommender Elemente}

\author{
Von B. Gy s a e und H. K or s ch ing
}

Kaiser-Wilhelm-Institut für Physik, Hechingen, und Max-Planck-Institut für Physik, Göttingen

(Z. Naturforschg. 2 a, 475 [1947]; eingeg. am 26. August 1947)

Ausgehend von einigen Literaturangaben ${ }^{1}$, die das Vorhandensein eines natürlichen, bisher nicht zugeordneten $\alpha$-Strahlers von etwa $2 \mathrm{~cm}$ Reichweite wahrscheinlich machen, haben wir uns auf Grund zahlreicher Messungen von der Existenz dieses Körpers überzeugt. Im Laufe unserer Untersuchungen gelang uns eine sehr weitgehende Anreicherung. Es stellte sich dabei das Vorhandensein von mindestens drei $\alpha$-Reichweiten heraus. Wir bestimmten die Reichweiten zu $1,2 \mathrm{~cm}, 1,9 \mathrm{~cm}$ und $2,2 \mathrm{~cm}$. Daß es sich bei diesen Reichweiten tatsächlich um $\alpha$-Strahlen und nicht um spontane Kernspaltung handelt, zeigten wir durch Vergleich der Ionisationsstärke mit bekannten $\alpha$-Strahlern; Kerntrümmer müßten eine sehr viel stärkere Ionisation hervorrufen.

An den angereicherten, von Uran und Thorium befreiten Präparaten haben wir nun, ungestört durch Mitreißeffekte, die chemischen Eigenschaften untersucht. Es ergab sich, daß,dieselben zu keinem der Elemente bis herauf zur Ordnungszahl 92 paßten. Wir stellten unter anderem fest: die Nichtfällbarkeit mit Schwefelwasserstoff in saurer Lösung, die Löslichkeit des Hydroxyd-Niederschlags mit Ammoncarbonat; ferner wurde mit Oxalsäure keine Fällung erhalten. Wir fanden aber auch Reaktionen, die es gestatteten, die drei Körper voneinander zu trennen, wodurch der Be- weis erbracht ist, daß die drei Strahler nicht Isotope eines Elementes sind. Ähnlich wie die Elemente 93 und $94^{2}$ ließen sich der 1,2- und der 2,2-cm-Strahler nach Reduktion mit Schwefeldioxyd mit Flußsäure fällen. Beim Oxydieren mit Ammoniumpersulfat trat kein Niederschlag auf.

Aus den gesamten chemischen Ergebnissen geht hervor, daß eine Zuordnung zu den Ordnungszahlen 1 bis 92 nicht möglich ist, so daß Ordnungszahlen größer als 92 wahrscheinlich sind. Es wäre danach möglich, daß diese $\alpha$-Strahler zu der Gruppe von Elementen gehören, bei denen die Elektronenhülle durch innere $5 \mathrm{f}$ Elektronen aufgefüllt wird. Als Hinweis darauf wären einige dem Thorium und Uran ähnliche Eigenschaften zu werten.

Betrachtet man aber andererseits die Folgerungen, die sich aus der Theorie des $\alpha$-Zerfalls ergeben, so folgt für die Annahme einer so hohen Ordnungszahl aus der Gamowschen Formel der Geiger-Nuttall-Beziehung - jedenfalls im Hinblick auf den 1,2-cm-Strahler -, daß dieses Element eine so lange Lebensdauer haben müßte, daß der Nachweis seiner Aktivität unmöglich wäre. Nur durch sehr spezielle Annahmen betreffs Kernradius, Kernform oder ähnliches wäre eine kürzere Lebensdauer denkbar.

Weitere Arbeiten sind im Gange, um eine eindeutige Zuordnung zu ermöglichen.

1 J. J o l y, Proc. Roy. Soc. [London] 102, 682 [1923]; H. Z i e g e r t, Z. Physik 46, 668 [1928]; J. Sch intlmeister, Anz. Akad. Wiss. Wien, math.-nat. Kl. 1935, 181 u. 1936, 2.

${ }^{2}$ E.M c M ill an u. P.H. A bel s o n, Physic. Rev. 57, 1185 [1940]; F. St r a $ß$ m an u. O. H a hn, Naturwiss. 30, 258 [1942]; G. T. S e a b o r g, A. C. W a h 1 u. I. W. Ke n n ed y, Physic. Rev. 69, 367 [1945].

\title{
BUCHBESPRECHUNGEN
}

Theorie der Supraleitung. Von M. v. La u e. SpringerVerlag, Berlin u. Göttingen 1947. 124 S. mit $31 \mathrm{Ab}-$ bildungen. Preis geh. RM. 12.-.

Die jahrelangen Bemühungen v. Laues, die Londonsche Theorie der Supraleitung zu einer in sich widerspruchslosen phänomenologischen Theorie zu erweitern, haben so schöne Erfolge gezeitigt, daß er jetzt eine Fülle von Ergebnissen in Form einer Monographie vorlegen kann. Nur ein verhältnismäßig kleiner Teil ihres Inhaltes ist den an der Supraleitung interessierten Kreisen aus Veröffentlichungen von Laues in den letzten Jahren bekannt geworden, alles andere hat er erst in jüngster Zeit erarbeitet. Um so erfreulicher ist es und es verschafft uns einen besonderen ästhetischen Genuß, daß wir sogleich die geschlossene Theorie in einheitlicher und eleganter Darstellung vorgeführt bekommen. Alle Widersprüche, die F.u.H.London in ihrer Elektrodynamik des Supraleiters selbst noch bedrückten, lösen sich hier aufs schönste; alle Unzulänglichkeiteñ, die den thermodynamischen Ableitungen Gorters und Londons noch anhafteten, sind beseitigt. Einige der experimentellen Ergebnisse, die bisher unverständlich schienen, wie etwa das Verhalten von Supraleitern gegenüber Hochfrequenzströmen, finden zwanglos ihre Erklärung. Allerdings handelt es sich, wie der Verfasser einleitend bemerkt, zunächst um eine für den idealen Einkristall gültige Theorie. Wir denken aber, daß, ebenso wie die Maxw ellsche Theorie zur Grundlage für die Elektrodynamik geworden ist, ehe die komplizierten Erscheinungen in ferromagnetischen Körpern erklärt worden waren, die v.Laue-Londonsche Theorie für die Elektrodynamik der Supraleiter als gültig anzusehen ist, ehe etwa die verwickelteren Er- 